Conclusion The risk of recurrence of GBS is reported between $1-6 \%$ (2) but recurrent MFS is extremely rare, with only 21 cases in the literature. In this case, the typical clinical presentation of MFS coupled with previous episodes may suggest an underlying predisposition, or even a pathogenic role for anti-gq1b antibody.

\title{
186 A CASE OF RECURRENT MILLER FISHER SYNDROME
}

Joy Thomas, Phil Smith, Ann Johnston. Department of Neurology, University Hospital Wales

\subsection{6/jnnp-2014-309236.186}

Introduction Miller Fisher Syndrome (MFS) is classically a postinfectious localized variant of Guillan-Barré Syndrome (GBS), composing a triad of ophthalmoplegia, ataxia and areflexia(1) although variants have been described. In light of this we outline an unusual case of recurrent MFS which challenges the recognised aetiology and/or points to an underlying predisposition.

Case A 56-year-old woman presented with subacute progressive ascending weakness, dysasthesia and dysarthria. On examination, there was complex ophthalmoplegia, bilateral lower motor neuron weakness in all limbs with dorsal column sensory loss to the hip and shoulder bilaterally. A clinical diagnosis of MFS was made and supported by nerve conduction studies and a strongly positive anti-gq1b antibody. Following treatment with intravenous immunoglobulin she made a full neurological recovery. However, she also reported two clinically similar episodes in 1980 and 1995 with full recovery, after which she was diagnosed with suspected relapsing remitting Multiple Sclerosis despite normal investigations. 